\title{
Long-Term Safety and Efficacy of Minimally Invasive Lumbar Decompression Procedure for the Treatment of Lumbar Spinal Stenosis With Neurogenic Claudication 2-Year Results of MiDAS ENCORE
}

\author{
Peter S. Staats, MD, MBA, * Timothy B. Chafin, MD, † Stanley Golovac, MD, $\neq$ Christopher K. Kim, MD, $\S$ \\ Sean Li, MD,// William B. Richardson, MD, ** Ricardo Vallejo, MD, PhD, †† Sayed E. Wahezi, MD, + t \\ Edward P. Washabaugh, III, MD, $\S \S$ and Ramsin M. Benyamin, MD $\dagger$ for the MiDAS ENCORE Investigators
}

\begin{abstract}
Background and Objectives: This study evaluated the long-term durability of the minimally invasive lumbar decompression (MILD) procedure in terms of functional improvement and pain reduction for patients with lumbar spinal stenosis and neurogenic claudication due to hypertrophic ligamentum flavum. This is a report of 2-year follow-up for MILD study patients.

Methods: This prospective, multicenter, randomized controlled clinical study compared outcomes for 143 patients treated with MILD versus 131 treated with epidural steroid injections. Follow-up occurred at 6 months and at 1 year for the randomized phase and at 2 years for MILD subjects only. Oswestry Disability Index, Numeric Pain Rating Scale, and Zurich Claudication Questionnaire were used to evaluate function and pain. Safety was evaluated by assessing incidence of device-/procedure-related adverse events.

Results: All outcome measures demonstrated clinically meaningful and statistically significant improvement from baseline through 6-month, 1-year, and 2-year follow-ups. At 2 years, Oswestry Disability Index improved by 22.7 points, Numeric Pain Rating Scale improved by 3.6 points, and Zurich Claudication Questionnaire symptom severity and physical function domains improved by 1.0 and 0.8 points, respectively. There were no serious device-/procedure-related adverse events, and $1.3 \%$ experienced a device-/procedure-related adverse event.
\end{abstract}

From the *National Spine and Pain Centers, Shrewsbury, NJ; †ंDepartment of Pain Management and Rehabilitation Medicine, Vidant Roanoke-Chowan Hospital, Ahoskie, NC; $\$$ Florida Pain Institute, Merritt Island, FL; §The Center for Pain Relief, Charleston, WV; |Premier Pain Centers, Shrewsbury, NJ; **Southeastern Spine Institute, Mount Pleasant, SC; †† Millennium Pain Center, Bloomington, IL; \$Departments of Physical Medicine and Rehabilitation and Anesthesiology, Albert Einstein College of Medicine at Montefiore, Montefiore Medical Center, Bronx, NY; and $\S$ Michigan Pain Specialists, Ypsilanti, MI.

Accepted for publication June 8, 2018.

Address correspondence to: Peter S. Staats, MD, MBA, National Spine and Pain Centers, 170 Avenue at the Common, Ste 6, Shrewsbury, NJ 07702 (e-mail: peterstaats@hotmail.com).

P.S.S. and R.M.B. are study principal investigators for MiDAS ENCORE. In this role, they have been responsible for study oversight. Responsibilities include protocol review, assistance with site selection, site investigator support, oversight of patient enrollment and protocol compliance, and adjudication of adverse events.

This trial was sponsored by Vertos Medical. The sponsorship includes studyrelated supplies and expenses, as well as funding for statistical analysis services by an independent provider

The authors declare no conflict of interest

Supplemental digital content is available for this article. Direct URL citations appear in the printed text and are provided in the HTML and PDF versions of this article on the journal's Web site (www.rapm.org).

Copyright (c) 2018 The Author(s). Published by Wolters Kluwer Health, Inc. on behalf of the American Society of Regional Anesthesia and Pain Medicine. This is an open-access article distributed under the terms of the Creative Commons Attribution-Non Commercial-No Derivatives License 4.0 (CCBY-NC-ND), where it is permissible to download and share the work provided it is properly cited. The work cannot be changed in any way or used commercially without permission from the journal.

ISSN: 1098-7339

DOI: 10.1097/AAP.0000000000000868
Conclusions: MILD showed excellent long-term durability, and there was no evidence of spinal instability through 2-year follow-up. Reoperation and spinal fracture rates are lower, and safety is higher for MILD versus other lumbar spine interventions, including interspinous spacers, surgical decompression, and spinal fusion. Given the minimally invasive nature of this procedure, its robust success rate, and durability of outcomes, MILD is an excellent choice for first-line therapy for select patients with central spinal stenosis suffering from neurogenic claudication symptoms with hypertrophic ligamentum flavum.

Clinical Trial Registration: This study was registered at ClinicalTrials. gov, identifier NCT02093520.

(Reg Anesth Pain Med 2018;43: 789-794)

$\mathrm{N}$ eurogenic claudication due to lumbar spinal stenosis (LSS) is associated with debilitating pain in the lower back and extremities and is the cause of significant functional limitation, especially in the elderly population. ${ }^{1}$ Neurogenic claudication symptoms are precipitated by walking and relieved by sitting. It is believed that spinal extension (walking and standing) produces neurogenic claudication symptoms by reducing the cross-sectional area of the central canal, resulting in nerve root compression and painful nerve root ischemia. This compression is relieved with spinal flexion, which causes the central canal to expand, leading to pain relief and resolution of neurogenic claudication symptoms. ${ }^{2,3}$ Unlike symptoms of radicular pain, the distribution of symptoms related to neurogenic claudication is not usually dermatomal. Radicular pain is related to inflammation of an affected nerve root and generally radiates from the back and buttock into the leg in a dermatomal pattern. ${ }^{4,5}$

Patients suffering from neurogenic claudication almost always present with degenerative soft tissue and bony pathology related to a combination of disc protrusion, thickened or ossified ligamentum flavum, facet joint hypertrophy, or osteophytes. ${ }^{1-3}$ In 1 report by Hansson and colleagues, ${ }^{3}$ ligamentum flavum hypertrophy (LFH) contributed to between $50 \%$ and $85 \%$ of central canal narrowing, leading the authors to conclude that the ligamentum flavum plays a dominant role in the load-induced narrowing of the lumbar spinal canal. Further, one of the common characteristics of neurogenic claudication is the high frequency of multiple-level stenosis. ${ }^{2,6}$

The MiDAS ENCORE study was approved by the Centers for Medicare \& Medicaid Services (CMS) as a Coverage with Evidence Development study for the purpose of providing level I evidence to support the safety and effectiveness of the MILD procedure. This randomized controlled trial assessed outcomes of the MILD procedure compared with epidural steroid injections (ESIs) in subjects with LSS and neurogenic claudication symptoms, 

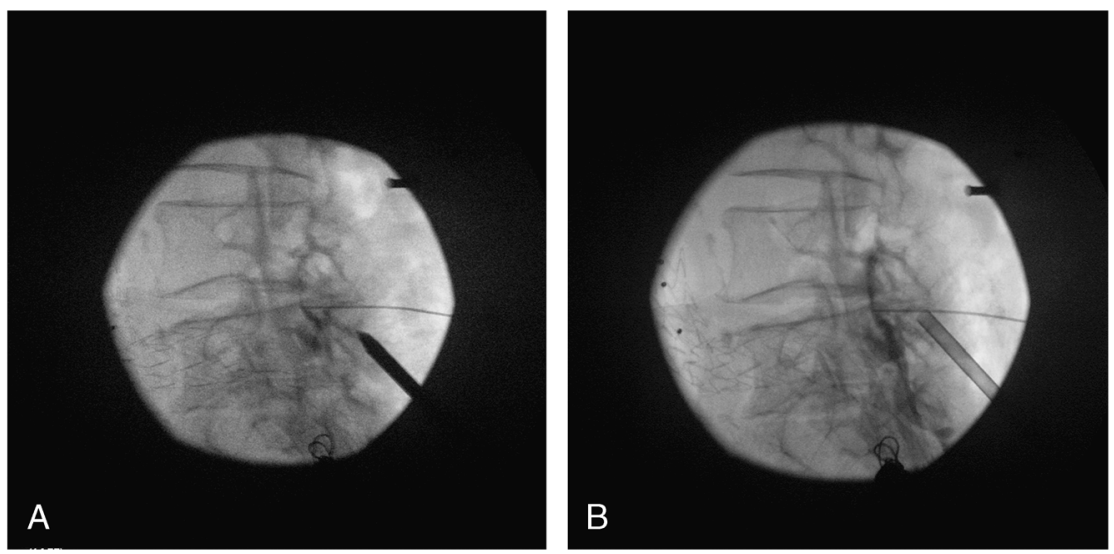

with verified LFH as a contributing factor. Study follow-up intervals occurred at 6 months and 1 year for the randomized phase of the study, and subjects in the MILD treatment group were followed at 2 years. Study design and 6-month and 1-year follow-up comparative results between MILD and ESI were previously reported. ${ }^{7-9}$ This is a report of 2-year clinical outcomes of patients treated with the MILD procedure in the MiDAS ENCORE study.

\section{METHODS}

This study was conducted at 26 interventional pain management centers throughout the United States. The trial protocol was approved by institutional review boards for all participating sites and registered with the US clinical trial registry (NCT02093520). Study patients were required to be 65 years or older and Medicare beneficiaries. Enrolled patients had central LSS with neurogenic claudication symptoms for at least 3 months and ligamentum flavum of greater than $2.5 \mathrm{~mm}$ in thickness. Subjects with Oswestry Disability Index (ODI) score of less than 31, Numeric Pain Rating Scale (NPRS) score of less than 5, prior surgery at any treatment level, or spondylolisthesis of grade III or higher were excluded from the study. The MiDAS ENCORE protocol was developed as a Coverage with Evidence Development study in collaboration with CMS. As designed and approved by CMS, the primary end point for the randomized phase of this study was 1-year follow-up. Twoyear outcome data were collected for patients in the MILD group only. An economic analysis demonstrating the cost-effectiveness of MILD versus other LSS therapies is the subject of a separate report that will be published subsequently.

MILD is a minimally invasive lumbar decompression procedure that is performed percutaneously through a $5.1-\mathrm{mm}$ port, thereby limiting trauma to tissue and bony structures of the spine. Generally using only moderate sedation and local anesthetic, the MILD procedure is conducted by accessing the interlaminar space from the posterior lumbar spine. Using the MILD devices together with contrast-enhanced fluoroscopic guidance, small amounts of lamina and hypertrophic ligamentum flavum are selectively removed, thereby achieving lumbar decompression. Fluoroscopic guidance is used throughout the MILD procedure, and visualization of the epidurogram contrast flow allows assessment of the amount of decompression. Following treatment with MILD, epidurogram contrast flow becomes thicker and straighter (Fig. 1). Once adequate decompression has been observed, all devices are removed, and the access site is usually closed with a sterile adhesive strip, with no sutures. The contralateral side and other levels may then be treated, as medically indicated. The MILD procedure leaves no implants behind and therefore preserves the option for patients to undergo subsequent more invasive treatment options that may require implants. The MILD procedure has been previously described.

Validated assessments including ODI, NPRS, and Zurich Claudication Questionnaire (ZCQ) were used to evaluate function and pain. Because neurogenic claudication symptoms are often intermittent, study patients were instructed to respond to these outcome assessments based on their experience over time as supported by

TABLE 1. MILD Patient Characteristics

\begin{tabular}{lc} 
Characteristic & MILD (n = 149) \\
\hline Age, ${ }^{*}$ y & $75.6 \pm 7.0$ \\
Gender & \\
Male & $49.7 \%(74)$ \\
Female & $50.3 \%(75)$ \\
Presenting LSS cofactors & \\
LFH & $100.0 \%(149)$ \\
Bulging disc & $89.9 \%(134)$ \\
Foraminal narrowing & $87.2 \%(130)$ \\
Facet hypertrophy & $86.6 \%(129)$ \\
Facet arthropathy & $76.5 \%(114)$ \\
Degenerative disc disease & $67.8 \%(101)$ \\
Disc space loss & $59.1 \%(88)$ \\
Lateral recess narrowing & $57.0 \%(85)$ \\
Osteophytes & $47.7 \%(71)$ \\
Spondylosis & $47.0 \%(70)$ \\
Spondylolisthesis & $44.3 \%(66)$ \\
Nerve root impingement & $33.6 \%(50)$ \\
Herniated disc & $27.5 \%(41)$ \\
Scoliosis & $22.1 \%(33)$ \\
Other & $19.5 \%(29)$ \\
ODI* & $53.0 \pm 12.9$ \\
NPRS* & $7.7 \pm 1.4$ \\
ZCQ* & \\
Symptom severity domain & $3.5 \pm 0.5$ \\
Physical function domain & $2.9 \pm 0.5$ \\
\hline
\end{tabular}

$*$ Mean \pm SD 
TABLE 2. Mean Change for MILD Efficacy Outcome Measures**

\begin{tabular}{lccc}
\hline $\begin{array}{l}\text { Outcome } \\
\text { Assessments* }\end{array}$ & $\begin{array}{c}\mathbf{6 - m o}(\mathbf{n}=\mathbf{1 3 3}) \text { Mean Improvement } \\
\mathbf{( 9 5 \%} \mathbf{C I})\end{array}$ & $\begin{array}{c}\mathbf{1 - y}(\mathbf{n}=\mathbf{1 1 9 )} \text { Mean Improvement } \\
\mathbf{( 9 5 \% ~ C I )}\end{array}$ & $\begin{array}{c}\mathbf{2 - y}(\mathbf{n}=\mathbf{9 9}) \dagger \text { Mean Improvement } \\
(\mathbf{9 5 \%} \mathbf{C I})\end{array}$ \\
\hline ODI & 20.4 & 19.5 & 22.7 \\
& $(17.1-23.7)$ & $(16.1-23.0)$ & $(18.5-26.9)$ \\
NPRS & 3.1 & 3.3 & 3.6 \\
& $(2.5-3.6)$ & $(2.8-3.9)$ & $(3.1-4.2)$ \\
ZCQ symptom & 0.8 & 0.9 & 1.0 \\
severity & $(0.7-1.0)$ & $(0.8-1.1)$ & $(0.8-1.2)$ \\
ZCQ physical & 0.7 & 0.6 & 0.8 \\
function & $(0.6-0.8)$ & $(0.5-0.8)$ & $(0.6-0.9)$ \\
\hline
\end{tabular}

*Clinically meaningful improvement: ODI $\geq 10$ points, NPRS $\geq 2.0, \mathrm{ZCQ} \geq 0.5$.

$\dagger$ ODI and ZCQ symptom severity consisted of 98 subjects completing the 2-year follow-up.

**Based on modified intent-to-treat statistical analysis.

validation studies. Clinically meaningful improvement in function was defined as equal to or greater than 10-point improvement in ODI score from baseline to follow-up. ${ }^{10,11}$ Clinically significant efficacy thresholds were defined as a 2-point improvement in NPRS, ${ }^{10-14}$ a 0.5 -point improvement in ZCQ domains, and an absolute ZCQ patient satisfaction score of 2.5 or less. ${ }^{15-17}$ Safety was evaluated by assessing incidence of device- or procedurerelated adverse events. Continuous data were tabulated with means and SDs, and categorical data were reported using frequency counts and percentages. For graphical displays, continuous data were reported using means and $95 \%$ confidence intervals (CIs). All $P$ values presented are 2 -sided, with $P<0.05$ considered significant.

\section{RESULTS}

One hundred forty-nine MILD patients were enrolled from June 2014 through April 2015. Of these, 6 subjects voluntarily withdrew prior to treatment, resulting in 143 MILD patients. Patient demographics and presenting LSS cofactors are presented in Table 1. Bulging disc, foraminal narrowing, facet hypertrophy, facet arthropathy, and degenerative disc disease were the most frequently reported presenting LSS cofactors. Ninety-five percent of patients presented with 5 or more spinal comorbidities. Baseline clinical presentation, ODI, NPRS, and ZCQ domains are also presented in Table 1.

At 2 years, a total of 26 patients had been withdrawn because of receipt of disallowed secondary intervention or study withdrawal with the intent to receive disallowed secondary intervention. The remaining 117 MILD patients were then potentially available for 2-year follow-up. Of those patients, 8 missed the 2-year follow-up visit, 5 withdrew for unrelated reasons, and 5 died of reasons unrelated to the MILD procedure including stroke and cardiac arrest. The remaining patients comprise the modified intent-to-treat population of 99 MILD patients who returned for 2-year follow-up. The modified intent-to-treat analysis includes all observed data for each follow-up visit reported. Subjects who missed a given follow-up, or who withdrew prior to that followup, are not included in the analysis for that visit.

At 2-year follow-up, all primary and secondary efficacy outcome measures showed clinically meaningful and statistically

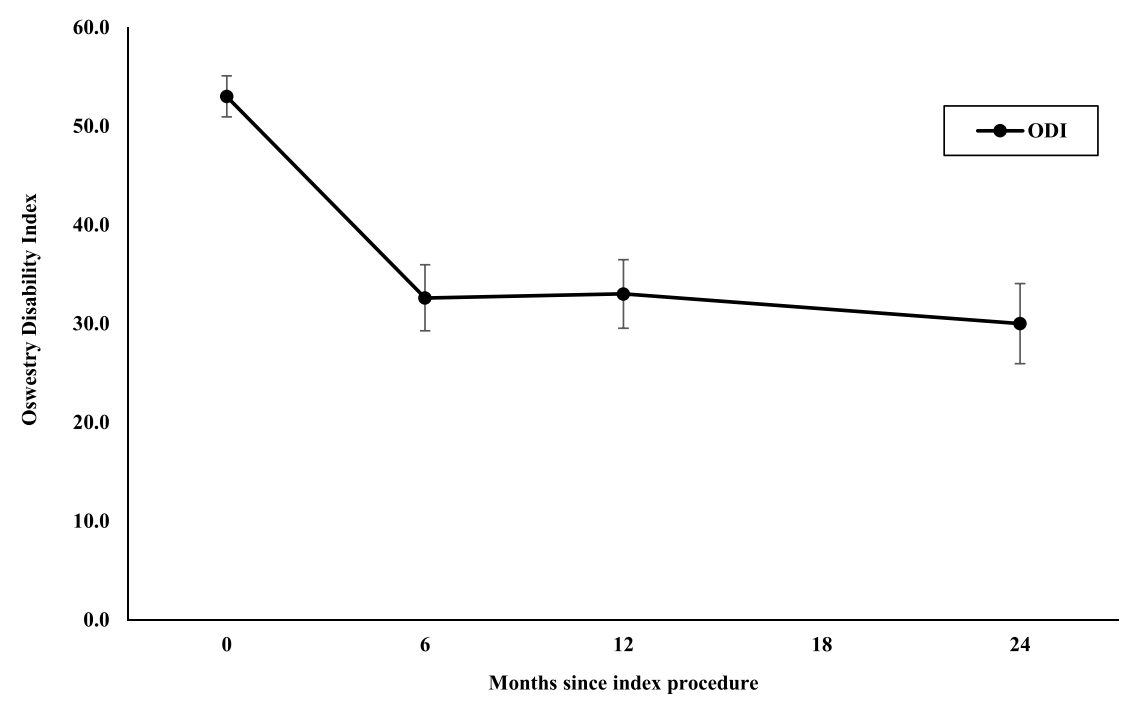

FIGURE 2. Oswestry Disability Index mean improvement at all follow-up intervals was clinically meaningful and statistically significant $(P<0.001)$ using modified intent-to-treat statistical analysis method. The modified intent-to-treat analysis includes all observed data for each follow-up visit reported. Subjects who missed a given follow-up, or who withdrew prior to that follow-up, are not included in the analysis for that visit. 


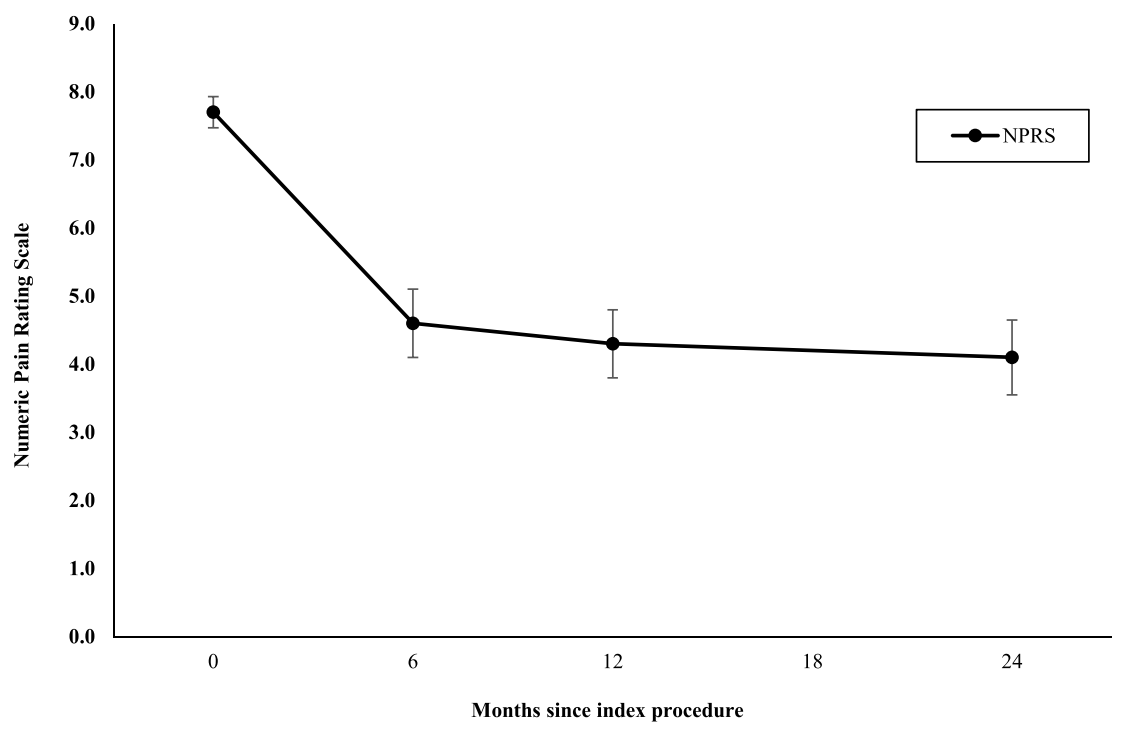

FIGURE 3. Numeric Pain Rating Scale mean improvement at all follow-up intervals was clinically meaningful and statistically significant $(P<0.001)$ using modified intent-to-treat statistical analysis method. The modified intent-to-treat analysis includes all observed data for each follow-up visit reported. Subjects who missed a given follow-up, or who withdrew prior to that follow-up, are not included in the analysis for that visit.

significant improvement from baseline and remained stable compared with 6-month and 1-year follow-ups. At 2 years, ODI improved by 22.7 points (95\% CI, 18.5-26.9), NPRS improved by 3.6 points $(95 \% \mathrm{CI}, 3.1-4.2)$, and ZCQ symptom severity and physical function domains improved by 1.0 (95\% CI, $0.8-1.2)$ and $0.8(95 \%$ CI, 0.6-0.9) points, respectively (Table 2$)$. Figure 2 shows mean ODI values at baseline and 6-month, 1-year, and 2-year follow-ups. Figures 3 and 4 show similar mean values for NPRS, ZCQ symptom severity, and ZCQ physical function at baseline and all follow-ups. Mean changes from baseline exceeded the clinically meaningful threshold and achieved statistical significance at $P<0.001$ for all efficacy end points and follow-up times. The ZCQ patient satisfaction score of 2.0 (95\% CI, 1.8-2.2) at 2-year follow-up exceeded the validated clinically meaningful threshold of 2.5 or less. At 2 years, responder rates for ODI, NPRS, and
ZCQ symptom severity, physical function and patient satisfaction were $72.4 \%, 71.7 \%, 73.5 \%, 59.6 \%$ and $76.8 \%$, respectively.

During 2-year follow-up, no MILD patients underwent a subsequent MILD procedure at any level. Eight (5.6\%) of 143 patients underwent a subsequent surgical procedure at the index level, 22 (15.4\%) of 143 received an ESI or nerve block at the level of surgery, and one of these patients also received a spinal cord stimulator as a treatment for the pain at the index level. One additional patient received a rhizotomy at the index level, and 1 patient received an intrathecal infusion pump (Table 3).

There were no serious device- or procedure-related adverse events reported for these patients, and there was no evidence of spinal instability at 2 years after the MILD procedure. As previously reported, 2 MILD patients (1.3\%) experienced a device- or procedure-related adverse event in this study, which was the same

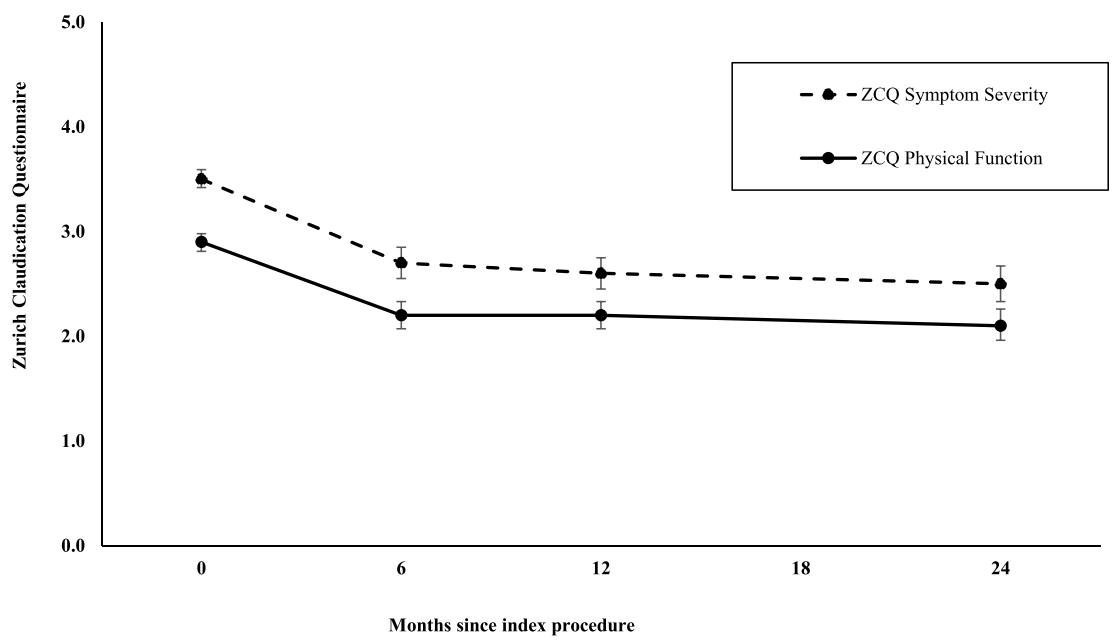

FIGURE 4. Mean improvement for ZCQ symptom severity and ZCQ physical function domains at all follow-up intervals was clinically meaningful and statistically significant $(P<0.001)$ using modified intent-to-treat statistical analysis method. The modified intent-to-treat analysis includes all observed data for each follow-up visit reported. Subjects who missed a given follow-up, or who withdrew prior to that follow-up, are not included in the analysis for that visit. 
TABLE 3. Reoperations and Retreatments Through 2-Year Follow-Up for MILD Patients

\begin{tabular}{lc}
\hline & MILD, n/N (\%) \\
\hline Surgical procedure & $8 / 143(5.6)$ \\
ESI or nerve block* & $22 / 143(15.4)$ \\
Rhizotomy & $1 / 143(0.7)$ \\
Intrathecal pump & $1 / 143(0.7)$ \\
\hline
\end{tabular}

*Includes 1 patient who also received a spinal cord stimulator.

rate as the ESI patients in the control arm during the randomized phase of this study $(P=1.00))^{8,9}$ During 1 MILD case, intraoperative oozing was observed at the decompression site, and Gelfoam was administered through the cannula into the interlaminar space. The patient was discharged on the same day as the procedure with no complications. A second patient experienced postoperative pain possibly related to MILD that resolved within 3 days of the index procedure.

\section{DISCUSSION}

Two-year follow-up results of this study demonstrate excellent durability of the MILD procedure for LSS patients suffering from neurogenic claudication symptoms and hypertrophic ligamentum flavum. All outcome measures demonstrated clinically meaningful and statistically significant improvement from baseline through 6-month, 1-year, and 2-year follow-ups. MILD patients experienced a mean ODI improvement of 22.7 points from baseline to 2 years postprocedure. This is markedly higher than the 10-point improvement that has been validated to be the threshold for clinically significant improvement for ODI. ${ }^{10,11}$ Further, $72.4 \%$ of MILD patients at 2 years achieved at least a 10-point improvement in ODI. All other validated efficacy measures also showed clinically meaningful and statistically significant improvement, including NPRS, ZCQ physical function, and ZCQ symptom severity (Table 2). Zurich Claudication Questionnaire patient satisfaction also exceeded the clinically meaningful threshold.

The overall surgical reoperation rate for MILD patients in this study was only $5.6 \%(8 / 143)$ at 2-year follow-up (Table 3 ). MILD has demonstrated an excellent safety profile with no device- or procedure-related serious adverse events, and a $1.3 \%$ rate of device- and procedure-related adverse events, which was similar to the rate for ESIs. ${ }^{9}$ Further, there was no evidence of spinal instability through 2-year follow-up.

As a benchmark, the rate of patients undergoing reoperations through 2 years after nonfusion decompression surgery was reported by the SPORT investigators to be $7.8 \% .{ }^{18}$ At 2 years, reoperation rates after spinal fusion have been reported to be $12.5 \%$ to $16.9 \%,{ }^{19-21}$ and after interspinous process distraction were $14.4 \%$ to $26.0 \%{ }^{22,23}$ Although this study did not provide a headto-head comparison to other LSS therapies, a discussion of safety outcomes for the broad range of LSS treatments provides important context related to the overall clinical management of LSS and effects on decision making regarding treatment algorithms for these patients. A randomized controlled investigational device exemption study comparing 2 spacers reported device-related adverse event rates of $11.6 \%$ and $7.5 \%$, and procedure-related adverse event rates of $14.2 \%$ and $15.9 \%$ through 2 -year follow-up. ${ }^{22}$ The SPORT investigators reported a $9.9 \%$ rate of intraoperative complications and a $12.3 \%$ rate of postoperative complications following surgical decompression through 2 years. ${ }^{18}$ Spinal fusion studies have reported related complication rates as high as $23.3 \%$ through 2 years. ${ }^{24,25}$ The rate of device removal at 2 -year follow-up has been reported to be $16.3 \%$ for a currently marketed IPD device. ${ }^{22}$ The rate of lumbar spine fracture has been reported to be $4.2 \%$ for fusion procedures ${ }^{21}$ and $16.3 \%$ for a currently marketed spacer $^{22}$ at 2-years.

The addition of MILD to the array of treatment options for spinal stenosis is valuable to treating physicians as well as patients. MILD is safe and has been shown to provide durable results through 2-year follow-up. MILD does not involve the use of implants, is performed as an outpatient procedure without general anesthesia, requires only a small $5.1-\mathrm{mm}$ port for access, and does not require sutures (although some surgeons elect to place a single suture at the portal site). In the comparative trial of MILD against ESIs, the success rate including durability was greater than ESIs, with a comparable safety profile. ${ }^{9}$ Given the minimally invasive nature of this procedure, its robust success rate, and durability of outcomes, MILD is an excellent choice for patients suffering from neurogenic claudication symptoms with LFH, who are refractory to conservative care.

While open surgery is often an excellent choice and may be required for certain patients, MILD can be offered for patients who may not tolerate a more invasive procedure, and it has a unique role as a solution when conservative therapies have failed, and the risks of more invasive approaches may not be warranted. It is important to note that MILD does not affect surgical options for the few patients who do not respond to this treatment. Because of the very minimally invasive approach and targeted subtle decompression, there is minimal or no scar tissue that would increase the risk of possible future open spine surgery. Another important consideration related to treatment planning is that patients undergoing open surgical decompression are no longer candidates for less invasive approaches.

The average age of patients in this study was 75.6 years, with patients as old as 93 years. This can be compared with an average age of 63.6 years for the surgical patients in SPORT ${ }^{18}$ and 66.9 and 66.2 years for the treatment and control arms, respectively, of the recent US Food and Drug Administration randomized controlled trial comparing safety and clinical outcomes of spacers. ${ }^{22}$ In addition, there was a high rate of presenting spinal comorbidities for MILD patients enrolled in this study (Table 1). MILD is intended for patients with central stenosis due to LFH; however, bulging discs, foraminal narrowing, and facet hypertrophy or arthrosis were common and actually were associated with higher rates of response than the ENCORE population as a whole (Table 4). Further, responder rates for patients with bulging disc and facet hypertrophy were statistically significantly higher than those without these comorbidities. Thus, our data suggest that

TABLE 4. ODI Response Rate by LSS Cofactor Subgroup at 2-Year Follow-Up

\begin{tabular}{llcl}
\hline Characteristic & Not Present & Present & $\boldsymbol{P}$ \\
\hline Bulging disc & $27.3 \%(3 / 11)$ & $77.3 \%(68 / 88)$ & $0.002 *$ \\
Foraminal narrowing & $60.0 \%(9 / 15)$ & $73.8 \%(62 / 84)$ & 0.351 \\
Facet hypertrophy & $47.1 \%(8 / 17)$ & $76.8 \%(63 / 82)$ & $0.019 *$ \\
Facet arthropathy & $68.2 \%(15 / 22)$ & $72.7 \%(56 / 77)$ & 0.789 \\
Degenerative disc disease & $65.5 \%(19 / 29)$ & $74.3 \%(52 / 70)$ & 0.463 \\
Disk space/height loss & $61.0 \%(25 / 41)$ & $79.3 \%(46 / 58)$ & 0.069 \\
Lateral recess narrowing & $65.0 \%(26 / 40)$ & $76.3 \%(45 / 59)$ & 0.259 \\
\hline
\end{tabular}

ODI responder defined as improvement of $\geq 10$ points from baseline.

*Statistically significant at $P<0.05$. 
the mere presence of these comorbidities should not be used as an exclusion.

The experience of MILD patients in this study is similar and compares favorably to 2-year MILD results previously reported. ${ }^{26}$ In the report by Chopko, ${ }^{26}$ patients demonstrated a statistically significant reduction of pain and statistically significant improvement in physical function and mobility as measured by ODI and all ZCQ domains, from baseline through 2 years. At 2 years, mean ZCQ patient satisfaction indicated that patients were satisfied with their procedure. In addition, no major device or procedural complications were reported.

The limitations of this study include lack of a control group at 2-year follow-up. The randomized controlled portion of the study concluded at the primary end point of 1 year, and supplementary follow-up through 2 years was conducted for the MILD patient group only. This study did not compare efficacy directly with open surgical approaches, including lumbar decompression, fusion, or spacers. Study limitations have been previously described. ${ }^{7-9}$

Two-year follow-up for MILD patients in this study showed excellent durability of the MILD procedure for treatment of LSS patients with neurogenic claudication and LFH. All efficacy outcomes achieved clinically meaningful and statistically significant improvement from baseline to all follow-up intervals through 2 years. There was no evidence of spinal instability at 2 years after the MILD procedure. A comparison of reoperation rates, spinal fractures, and safety between MILD and the broad range of lumbar spine interventions, including spacers, surgical decompression, and spinal fusion, shows a dramatically lower rate for MILD in all categories. These results confirm the importance of this therapy, and support MILD's position as an optimal interventional option for patients suffering from neurogenic claudication symptoms with hypertrophic ligamentum flavum.

\section{ACKNOWLEDGMENTS}

The authors thank the MiDAS ENCORE Investigators for their valuable contributions to this study (see the Appendix, Supplemental Digital Content 1, http://links.lww.com/AAP/A275, for a listing). They also thank Scott Brown, PhD, of Altair Biostatistics LLC for statistical analysis and the editorial board of Regional Anesthesia and Pain Medicine for review of this manuscript.

\section{REFERENCES}

1. Katz JN, Harris MB. Lumbar spinal stenosis. N Engl J Med. 2008;358: 818-825.

2. Porter RW. Spinal stenosis and neurogenic claudication. Spine. 1996;21: 2016-2052

3. Hansson T, Suzuki N, Hebelka H, Gaulitz A. The narrowing of the lumbar spinal canal during loaded MRI: the effects of the disc and ligamentum flavum. Eur Spine J. 2009; 18:679-686.

4. Allegri M, Montella S, Salici F, et al. Mechanisms of low back pain: a guide for diagnosis and therapy [version 2]. F1000Res. 2016;5: F1000 Faculty Rev-1530.

5. Bogduk N. On the definitions and physiology of back pain, referred pain, and radicular pain. Pain. 2009;147:17-19.

6. Mekhail N, Costandi S, Abraham B, Samuel SW. Functional and patient-reported outcomes in symptomatic lumbar spinal stenosis following percutaneous decompression. Pain Pract. 2012;12:417-425.

7. Benyamin RM, Staats PS. MiDAS ENCORE: randomized controlled study design and protocol. Pain Physician. 2015;18:307-316.
8. Staats PS, Benyamin RM. MiDAS ENCORE: randomized controlled clinical trial report of 6-month results. Pain Physician. 2016;19:25-37.

9. Benyamin RM, Staats PS. MILD ${ }^{\circledR}$ is an effective treatment for lumbar spinal stenosis with neurogenic claudication: MiDAS ENCORE randomized controlled trial. Pain Physician. 2016;19:229-242.

10. Hägg O, Fritzell P, Nordwall A, Swedish Lumbar Spine Study Group. The clinical importance of changes in outcome scores after treatment for chronic low back pain. Eur Spine J. 2003;12:12-20.

11. Ostelo RWJG, Deyo RA, Stratford P, et al. Interpreting change scores for pain and functional status in low back pain towards international consensus regarding minimal important change. Spine. 2008;33:90-94.

12. Childs JD, Piva SR, Fritz JM. Responsiveness of the numeric pain rating scale in patients with low back pain. Spine. 2005;30:1331-1334.

13. Farrar JT, Young JP Jr, LaMoreaux L, Werth JL, Poole RM. Clinical importance of changes in chronic pain intensity measured on an 11-point numerical pain rating scale. Pain. 2001;94:149-158

14. Salaffi F, Stancati A, Silvestri CA, Ciapetti A, Grassi W. Minimal clinically important changes in chronic musculoskeletal pain intensity measured on a numerical rating scale. Eur J Pain. 2004;8:283-291.

15. Stucki G, Liang MH, Fossel AH, Katz JN. Relative responsiveness of condition-specific and generic health status measures in degenerative lumbar spinal stenosis. J Clin Epidemiol. 1995;48:1369-1378.

16. Stucki G, Daltroy L, Liang MH, Lipson SJ, Fossel AH, Katz JN. Measurement properties of a self-administered outcome measure in lumbar spinal stenosis. Spine. 1996;21:796-803.

17. Zucherman JF, Hsu KY, Hartjen CA, et al. A prospective randomized multi-center study for the treatment of lumbar spinal stenosis with the $\mathrm{X}$ STOP interspinous implant: 1-year results. Eur Spine J. 2004;13:22-31.

18. Weinstein JN, Tosteson TD, Lurie JD, et al. Surgical versus nonsurgical therapy for lumbar spinal stenosis. $N$ Engl J Med. 2008;358:794-810.

19. Irmola TM, Hakkinen A, Jarvenpaa S, Marttinen I, Vihtonen K, Neva M Reoperation rates following instrumented lumbar spine fusion. Spine. 2018;43:295-301.

20. Forsth P, Olafsson G, Carlsson T, et al. A randomized, controlled trial of fusion surgery for lumbar spinal stenosis. $N$ Engl J Med. 2016;374: 1413-1423.

21. Ong KL, Auerbach JD, Lau E, Schmier J, Ochoa JA. Perioperative outcomes, complications, and costs associated with lumbar spinal fusion in older patients with spinal stenosis and spondylolisthesis. Neurosurg Focus. 2014;36:E5.

22. Food and Drug Administration (US) [Internet]. Superion ${ }^{\circledR}$ InterSpinous Spacer Summary of Safety \& Effectiveness Data. Silver Spring, MD: CDRH; 2015: Available at: https://www.accessdata.fda.gov/cdrh_docs/ pdf14/P140004b.pdf. Accessed November 6, 2017.

23. Strömqvist BH, Berg S, Gerdhem P, et al. X-Stop versus decompressive surgery for lumbar neurogenic intermittent claudication: randomized controlled trial with 2-year follow-up. Spine. 2013;38:1436-1442.

24. Choi JM, Choi MK, Kim SB. Perioperative results and complications after posterior lumbar interbody fusion for spinal stenosis in geriatric patients over than 70 years old. J Korean Neurosurg Soc. 2017;60: 684-690.

25. Fritzell P, Hägg O, Wessberg P, Nordwall A. 2001 Volvo Award Winner in Clinical Studies: lumbar fusion versus nonsurgical treatment for chronic low back pain. Spine. 2001;26:2521-2534

26. Chopko BW. Long-term results of percutaneous lumbar decompression for LSS: two-year outcomes. Clin J Pain. 2013;29:939-943. 